\title{
Analytical Evaluation of the Static MoM Terms for Volume and Surface Rectangular Domains
}

\author{
Francisco J. Pérez Soler, Associate Member, IEEE, Fernando Daniel Quesada Pereira, Member, IEEE, \\ Alejandro Alvarez Melcon, Senior Member, IEEE, Benito Gimeno, Member, IEEE, \\ Vicente E. Boria Esbert, Senior Member, IEEE, and Luca Perregrini, Senior Member, IEEE
}

\begin{abstract}
We present a simple analytical implementation of the static singular integrals arising in the volume/surface integral equation formulation for the analysis of mixed conducting and dielectric structures such as printed circuits with finite-size dielectric objects. The singularity of the Green's functions is extracted from the kernel of the integral equation as a static term. This term is then evaluated analytically for coincident rectangular cells in two and three dimensions. The new technique allows an accurate and efficient evaluation of method of moments (MoM) self-interactions compared to other methods previously used in the literature.
\end{abstract}

Index Terms-Dielectrics, electromagnetic analysis, integral equations, integration, moment methods.

\section{INTRODUCTION}

$\mathbf{T}$ HE volume integral equation technique (VIE) is a widely used formulation for the analysis of a large class of problems containing dielectric objects of finite dimensions and complex shapes [1]. In this kind of problem, Green's functions cannot account for the dielectric objects, and one has to resort to the use of the well-known free-space Green's functions [2]. Using this formulation, the dielectric objects are modeled with the aid of polarization currents defined on their volumes. The subsequent application of the method of moments (MoM) requires the computation of overlapping integrals between the free-space Green's functions and the basis and testing functions (base and test integrals). When developing this approach, surface charge distributions appear at the outer surfaces of homogeneous bodies. As a result, several combinations of surface and volume integrals also need to be computed.

In the above frame, the authors have derived an efficient implementation of the volume/surface integral equation (VSIE) approach for the analysis of mixed dielectric and metallic objects. The efficiency of the formulation is increased by following

Manuscript received January 22, 2010. Date of publication February 22, 2010; date of current version March 12, 2010. This work was supported by SENECA Project Ref. 08833/PI/08 and CICYT Project Ref. TEC2007-67630-C03.

F. J. Pérez Soler, F. D. Quesada Pereira, and A. Alvarez Melcon are with the Departamento de Tecnologías de la Información y las Comunicaciones, Universidad Politécnica de Cartagena, 30202 Cartagena, Spain (e-mail: fernando.quesada@upct.es).

B. Gimeno is with the Departamento de Física Aplicada-ICMUV, Universitat de València, 46100 Burjassot, Spain.

V. E. Boria Esbert is with the Departamento de Comunicaciones-iTEAM, Universidad Politécnica de Valencia, Camino de Vera s/n, 46022 Valencia, Spain.

L. Perregrini is with the Electronic Department, University of Pavia, 27100 Pavia, Italy.

Digital Object Identifier 10.1109/LAWP.2010.2043710 a singularity extraction technique combined with analytical integration. The free-space Green's functions are first split into static and dynamic parts. The dynamic part thus obtained is bounded, and it can be integrated numerically without effort. What remains then is the calculation of the static term containing the singular behavior of the Kernel. To perform this task, integration techniques based on transformations from rectangular to cylindrical or spherical coordinates have been developed in the past [3]. Other approaches to treat this problem are based on the analytical integration of the singular term of the Kernel [4].

In this letter, we also propose to apply analytical integration to the singular term. However, as a novelty with respect to other previous works [4], we apply analytical integration at the same time to both source and observation cells. In this way, both test and base integrals are evaluated analytically. The proposed analytical integration increases accuracy and reduces considerably the computational cost for medium-size problems. The analytical integration is based on the dyadic identities reported in [5], which are extended for the first time to account for volume integration and mixed surface-volume interactions with rectangular domains in two and three dimensions. The included results show the efficiency and accuracy of the new approach for the treatment of the singularity in comparison to other previous techniques [3], [4]. This novel integration technique is of practical interest for the analysis of microstrip antennas or radomes.

\section{THEORY}

The formulation under consideration is a general VSIE technique including metallic and dielectric objects. First, the static term of the Green's functions is extracted following a Taylor series expansion. A standard VSIE formulation leads to the following source and volume integrals for the static term:

$$
\begin{aligned}
I_{1} & =\int_{\mathrm{S}} \int_{\mathrm{S}^{\prime}} \frac{1}{R_{\mathrm{S}}} d S^{\prime} d S ; \quad \text { on metallic surfaces } \\
I_{2} & =\int_{\mathrm{S}} \vec{f}_{\mathrm{t}} \cdot \int_{\mathrm{S}^{\prime}} \frac{1}{R_{\mathrm{S}}} \vec{f}_{\mathrm{b}} d S^{\prime} d S ; \quad \text { on metallic surfaces } \\
I_{3} & =\int_{\mathrm{V}} \int_{\mathrm{V}^{\prime}} \frac{1}{R_{\mathrm{V}}} d V^{\prime} d V ; \quad \text { in dielectric objects } \\
I_{4} & =\int_{\mathrm{V}} \vec{f}_{\mathrm{t}} \cdot \int_{\mathrm{V}^{\prime}} \frac{1}{R_{\mathrm{V}}} \vec{f}_{\mathrm{b}} d V^{\prime} d V ; \quad \text { in dielectric objects } \\
I_{5} & =\int_{\mathrm{V}} \int_{\mathrm{S}^{\prime}} \frac{1}{R_{\mathrm{VS}}} d S^{\prime} d V ; \quad \text { on the dielectric surfaces }
\end{aligned}
$$

where $\vec{f}_{\mathrm{b}}$ and $\vec{f}_{\mathrm{t}}$ are the basis and testing functions employed in the MoM implementation. Also, $R_{\mathrm{S}}$ is the spatial distance 


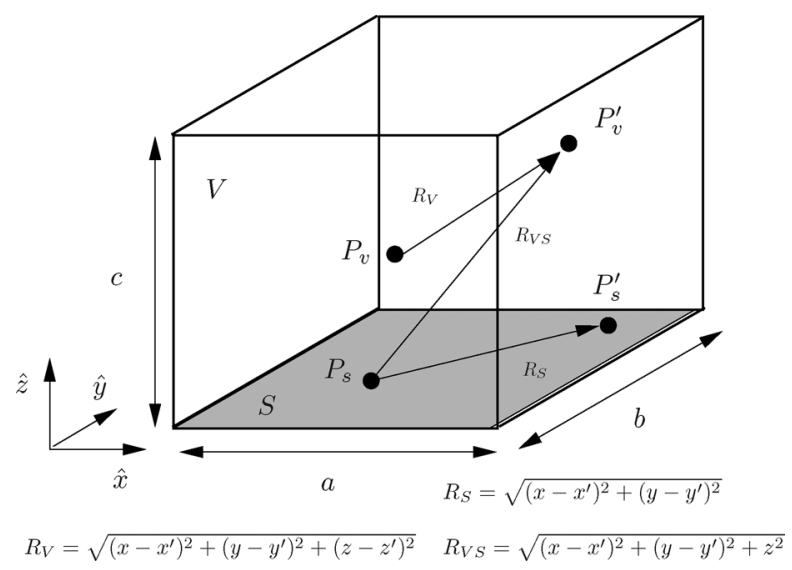

Fig. 1. General geometry of the integration domains considered in this letter.

between observation and source points considering two dimensions (surface case), whereas $R_{\mathrm{V}}$ is the volumetric counterpart of this distance. Finally, $R_{\mathrm{VS}}$ denotes a distance between a point inside a volumetric region and another point placed at one of the surfaces that encloses that region (see Fig. 1). Integrals (1) and (3) are those corresponding to the static MoM contributions of the electric scalar potential in two and three dimensions, respectively. On the other hand, (2) and (4) refer to the contributions of the magnetic vector potential in the same cases. Finally, (5) corresponds to a mixed surface-volume interaction due to the external charges distributed in the outer surfaces of homogeneous bodies.

Integrals $I_{1}$ to $I_{5}$ can be performed numerically using standard quadrature and cubature rules [6], when source and observation integration domains do not coincide. For the coincident cases $S=S^{\prime}$ and $V=V^{\prime}$, a different strategy must be adopted in order to avoid numerical problems and serious inaccuracies. A useful approach consists in transforming the source and observation points into cylindrical or spherical coordinates. It is well known that the term $1 / R$ is canceled out mathematically due to the Jacobian that appears in these transformations. Alternatively, other authors have derived analytical expressions for the base integration [4]. The analytical integration absorbs the singularity of the Green's functions, so numerical integration can be applied to the outer test integration. In this letter, a new strategy is proposed based on a double analytical integration of terms of the form $1 / R$ for coincident surface and volume domains. In this way, no numerical integration is needed for the test integration. The procedure for triangular surfaces was presented in [5]. Based on this work, the authors have extended such procedure to rectangular domains for both surface and volume cases (as illustrated in Fig. 1).

\section{A. Identities and Integrals for Rectangular Domains}

In [5], the authors started from a set of useful algebraic identities that helped to reduce the complexity of the MoM integrals. In two dimensions, and adapted for rectangular surfaces, these identities take now the following form:

$$
\frac{1}{R_{\mathrm{S}}}=-\nabla_{\mathrm{S}} \cdot \nabla_{\mathrm{S}}^{\prime} R_{\mathrm{S}}
$$

$$
\begin{aligned}
\frac{\overline{\bar{I}}}{R_{\mathrm{S}}}= & \nabla_{\mathrm{S}} \times \nabla_{\mathrm{S}}^{\prime} \times \overline{\bar{I}} R_{\mathrm{S}}-\nabla_{\mathrm{S}} \nabla_{\mathrm{S}}^{\prime} R_{\mathrm{S}} \\
\left(\nabla_{\mathrm{S}}^{\prime} \times \overline{\bar{I}} \cdot \vec{f}_{\mathrm{b}} R_{\mathrm{S}}\right)= & \nabla_{\mathrm{S}}^{\prime} \times\left(R_{\mathrm{S}} \overrightarrow{\mathrm{f}}_{\mathrm{b}}\right) \\
\vec{f}_{\mathrm{t}} \cdot\left(\nabla_{\mathrm{S}} \nabla_{\mathrm{S}}^{\prime} R_{\mathrm{S}}\right) \cdot \vec{f}_{\mathrm{b}}= & \nabla_{\mathrm{S}} \cdot\left(\nabla _ { \mathrm { S } } ^ { \prime } \cdot R _ { \mathrm { S } } \left[\vec{f}_{\mathrm{t}} \vec{f}_{\mathrm{b}}\right.\right. \\
& \left.\left.+\frac{1}{3}\left(\vec{f}_{\mathrm{t}} \vec{R}_{\mathrm{S}}-\vec{R}_{\mathrm{S}} \overrightarrow{\mathrm{f}}_{\mathrm{b}}\right)-\frac{1}{9} \overline{\bar{I}} R_{\mathrm{S}}^{2}\right]\right) .
\end{aligned}
$$

Here, the subscript $\mathrm{S}$ indicates that the spatial distance and the differential operators are expressed in two-dimensional coordinates (Fig. 1). These are the same expressions that appear in [5] for triangular domains and Rao-Wilton-Glisson (RWG) basis and testing functions, except for the case of (9), where the constants $1 / 3$ and $1 / 9$ change depending on the definition of the basis functions employed (which are rooftops defined on rectangular cells for our case).

These identities can now be extended to the 3D case of rectangular prisms cells, resulting in

$$
\begin{aligned}
\frac{2}{R_{\mathrm{V}}} & =-\nabla_{\mathrm{V}} \cdot \nabla_{\mathrm{V}}^{\prime} R_{\mathrm{V}} \\
\frac{2 \overline{\bar{I}}}{R_{\mathrm{V}}} & =\nabla_{\mathrm{V}} \times \nabla_{\mathrm{V}}^{\prime} \times \overline{\bar{I}} R_{\mathrm{V}}-\nabla_{\mathrm{V}} \nabla_{\mathrm{V}}^{\prime} R_{\mathrm{V}} \\
\left(\nabla_{\mathrm{V}}^{\prime} \times \overline{\bar{I}} \cdot \vec{f}_{\mathrm{b}} R_{\mathrm{V}}\right) & =\nabla_{\mathrm{V}}^{\prime} \times\left(R_{\mathrm{V}} \vec{f}_{\mathrm{b}}\right) \\
\vec{f}_{\mathrm{t}} \cdot\left(\nabla_{\mathrm{V}} \nabla_{\mathrm{V}}^{\prime} R_{\mathrm{V}}\right) \cdot \vec{f}_{\mathrm{b}} & =\nabla_{\mathrm{V}} \cdot\left(\nabla _ { \mathrm { V } } ^ { \prime } \cdot R _ { \mathrm { V } } \left[\vec{f}_{\mathrm{t}} \vec{f}_{\mathrm{b}}+\frac{1}{4}\left(\vec{f}_{\mathrm{t}} \vec{R}_{\mathrm{S}}\right.\right.\right. \\
& \left.\left.\left.-\vec{R}_{\mathrm{S}} \vec{f}_{\mathrm{b}}\right)-\frac{1}{12} \overline{\bar{I}} R_{\mathrm{V}}^{2}\right]\right) .
\end{aligned}
$$

Now, the subscript $\mathrm{V}$ denotes that operations are performed in three dimensions (illustrated in Fig. 1). It can be noticed that these new identities maintain the same form as the previous ones, differing only in some constants that appear due to the additional spatial dimension.

Finally, because we are also interested in mixed surface-volume interactions, an additional identity must be added to complete the whole set of useful equations, namely

$$
\frac{2}{R_{\mathrm{VS}}}=-\nabla_{\mathrm{S}} \cdot \nabla_{\mathrm{S}}^{\prime} R_{\mathrm{VS}}+\frac{\partial^{2}}{\partial z^{2}} R_{\mathrm{VS}} .
$$

Using these identities in combination with the well-known Gauss, Stokes, and Curl theorems, the integrals expressed in (1)-(5) can be transformed into the following bounded integrand expressions:

$$
\begin{aligned}
& I_{1}=-\int_{\mathrm{C}} \int_{\mathrm{C}^{\prime}} R_{\mathrm{S}} \vec{u} \cdot \vec{u}^{\prime} d l^{\prime} d l \\
& \begin{aligned}
I_{2}=-\int_{\mathrm{C}} \int_{\mathrm{C}^{\prime}} R_{\mathrm{S}} \vec{u} \cdot & {\left[\vec{f}_{\mathrm{t}} \vec{f}_{\mathrm{b}}-\vec{f}_{\mathrm{b}} \vec{f}_{\mathrm{t}}+\frac{1}{3}\left(\vec{f}_{\mathrm{t}} \vec{R}_{\mathrm{S}}-\vec{R}_{\mathrm{S}} \vec{f}_{\mathrm{b}}\right)\right.} \\
& \left.+\overline{\bar{I}}\left(\vec{f}_{\mathrm{t}} \cdot \vec{f}_{\mathrm{b}}-\frac{1}{9} R_{\mathrm{S}}\right)\right] \cdot \vec{u}^{\prime} d l^{\prime} d l
\end{aligned} \\
& I_{3}=-\frac{1}{2} \int_{\mathrm{S}} \int_{\mathrm{S}^{\prime}} R_{\mathrm{V}} \vec{u} \cdot \vec{u}^{\prime} d S^{\prime} d S
\end{aligned}
$$




$$
\begin{aligned}
& I_{4}=-\frac{1}{2} \int_{\mathrm{S}} \int_{\mathrm{S}^{\prime}} R_{\mathrm{V}} \vec{u} \cdot {\left[\vec{f}_{\mathrm{t}} \vec{f}_{\mathrm{b}}-\vec{f}_{\mathrm{b}} \vec{f}_{\mathrm{t}}+\frac{1}{4}\left(\vec{f}_{\mathrm{t}} \vec{R}_{\mathrm{V}}-\vec{R}_{\mathrm{V}} \vec{f}_{\mathrm{b}}\right)\right.} \\
&\left.+\overline{\bar{I}}\left(\vec{f}_{\mathrm{t}} \cdot \vec{f}_{\mathrm{b}}-\frac{1}{12} R_{\mathrm{V}}\right)\right] \cdot \vec{u}^{\prime} d l^{\prime} d l \\
& I_{5}=\frac{1}{2}\left(-\int_{0}^{c} \int_{\mathrm{C}} \int_{\mathrm{C}^{\prime}} R_{\mathrm{VS}} \vec{u} \cdot \vec{u}^{\prime} d l^{\prime} d l d z\right. \\
&\left.\quad+\int_{\mathrm{S}} \int_{\mathrm{S}^{\prime}} \frac{c}{\left.R_{\mathrm{V}}\right|_{\left(z-z^{\prime}\right)=c}} d S^{\prime} d S\right) .
\end{aligned}
$$

In the above expressions, $\mathrm{C}$ and $\mathrm{C}^{\prime}$ denote the linear contours of the surface cells, and $\mathrm{S}$ and $\mathrm{S}^{\prime}$ correspond to the surface boundaries of the volume cells. The vectors $\left(\vec{u}, \vec{u}^{\prime}\right)$ are the outward vectors normal to the observation and source integration domains (linear or surface depending on the case), respectively. It is interesting to note that, due to the application of the identities, the term $1 / R$ has changed to $R$, which is bounded for $R \rightarrow 0$. Also, the complexity of the integration domains has been reduced in one dimension for each case. Hence, it is possible to evaluate analytically the expressions (15)-(19).

Although the integration domains are simple, the final analytical results for all the integrals require very long calculus and algebraic manipulations, specially in the case of the volumetric cells. Nevertheless, we have verified that these operations can be performed with well-known symbolic analysis software, such as Mathematica [7]. The resulting expressions are composed of simple logarithmic and inverse of trigonometric functions, depending only on the size cell dimensions. Therefore, they can be evaluated very quickly with a computer. The final expressions for rectangular domains $\left(I_{1}, I_{2}\right)$ and for volume-surface domains $\left(I_{5}\right)$ are included in the Appendix, whereas the formulas for volume domains have been published in a Web page [8] due to space reasons.

\section{RESULTS}

In this section, we propose to evaluate the performance of two different numerical techniques for the computation of the different static singular integrals, taking the analytical results derived in this letter as the reference results. The first technique is the semianalytical method proposed in [4], widely employed in the computation of the singular and close to singular interactions of the MoM. In this case, the base integral is reduced to line integrals along the edges of a polygon or polyhedron. These line integrals can be written in close form. Nevertheless, this technique requires a numerical integration for the test integral, which increases the computational cost when using a Galerkin MoM procedure. Moreover, this method yields to numerical inaccuracies when computing the integrals for observation points close to the edges of the source domain.

The second technique was presented in [3] and is based on a transformation from rectangular coordinates to cylindrical or spherical coordinates depending on the original integration domain. This procedure needs to perform a different coordinate transformation for each observation cubature point of the test integration. Once the coordinate transformation is done for each observation point, a Gauss-Legendre quadrature rule is employed for computing the base integral since the singularity has been canceled out by the Jacobian of the transformation.
TABLE I

NumBer OF INTEGRATION POINTS NEEDED IN THE TEST INTEGRAL FOR THE DIFFERENT STATIC SINGULAR INTEGRALS IN ORDER TO OBTAIN A RELATIVE ERROR BELOW 10-3

\begin{tabular}{|c||c|c|}
\hline- & Semianalytic - Wilton & Sph-Cyl coordinates \\
\hline \hline$I_{1}$ & 13 & 10 \\
\hline$I_{2}$ & 13 & 16 \\
\hline$I_{3}$ & 31 & 31 \\
\hline$I_{4}$ & 31 & 58 \\
\hline$I_{5}$ & 6 & 6 \\
\hline
\end{tabular}

Using this technique, the base integral is done in spherical or cylindrical coordinates, while the test integral is solved using cubature rules [6]. For the numerical tests, a standard rectangular (surface case) or hexahedral (volume case) cell of aspect ratio $(a ; b=0.5 a ; c=a)$ has been selected. In Table I, we present the number of integration points needed in the test integral for the two mentioned numerical techniques in order to obtain a relative error below $10^{-3}$. The computation of the relative errors has been done taking as the reference results the analytical expressions derived in this letter. For the second technique, we have selected 10 fixed points for integration along the three spherical coordinates. The test integral has been solved in all cases with specialized cubature rules derived from [6]. Results show that the mixed volume-surface integral $I_{5}$ requires the least number of integration points. This is because the singularity is concentrated in one extreme of the integration domain. On the contrary, the volume integral with linear variation $I_{4}$ requires more numbers of points than the others. This is because more points are needed to properly represent the volume region. These results give an idea on the gain in efficiency that can be obtained when the new analytical technique presented in this letter is used instead of the alternative numerical approaches.

\section{CONCLUSION}

In this letter, we have presented a simple and efficient technique for evaluating the singular contributions in the general frame of a volume/surface integral equation (VSIE) formulation solved by MoM. This formulation is very useful for the analysis of finite-size microstrip antennas, microstrip transmission lines with complex features, or antennas covered by radomes. A novel analytical integration in rectangular domains is applied to the static terms of the Green's functions (for both surface and volume cases). It is shown that the new technique improves the accuracy and reduces the computational cost, as compared to pure numerical or semianalytical integration. Useful numerical results are presented to confirm the practical value of the new approach.

\section{APPENDIX \\ ANALYTICAL EXPRESSIONS}

Considering the domains and the reference coordinate systems of Fig. 1, the basis and testing functions employed for the integrals are defined as a linear variation along the $\hat{x}$-axis. There are two possible orientation cases, one along positive $\hat{x}$-axis $\left(\vec{f}_{1}\right)$ and one along negative $\hat{x}$-axis $\left(\overrightarrow{f_{2}}\right)$, taking the following forms:

$$
\begin{aligned}
& \overrightarrow{f_{1}}=x \cdot \hat{x} \\
& \overrightarrow{f_{2}}=(a-x) \cdot(-\hat{x}) ; \quad 0 \leq x \leq a .
\end{aligned}
$$


Under these considerations, the surface-surface integral for the scalar potential is

$$
\begin{aligned}
I_{1}= & \frac{2}{3}\left(a^{3}+b^{3}\right)-\frac{2}{3}\left(a^{2}+b^{2}\right)^{3 / 2}-a b^{2} \ln \left(\frac{-a+\sqrt{a^{2}+b^{2}}}{a+\sqrt{a^{2}+b^{2}}}\right) \\
& -a^{2} b \ln \left(\frac{-b+\sqrt{a^{2}+b^{2}}}{b+\sqrt{a^{2}+b^{2}}}\right) .
\end{aligned}
$$

The surface-surface integral with linear variation takes different forms if the basis and test functions have equal or opposite orientations. When they have the same orientation, the following expression is obtained:

$$
\begin{gathered}
I_{2 a}=\frac{1}{180}\left\{24 a^{5}+60 a^{2} b^{3}+4 b^{5}-\left(24 a^{4}+4 b^{4}+58 a^{2} b^{2}\right)\right. \\
\sqrt{a^{2}+b^{2}}+60 a^{3} b^{2} \ln \left(\frac{a+\sqrt{a^{2}+b^{2}}}{-a+\sqrt{a^{2}+b^{2}}}\right) \\
\left.+45 a^{4} b \ln \left(\frac{b+\sqrt{a^{2}+b^{2}}}{-b+\sqrt{a^{2}+b^{2}}}\right)\right\} .
\end{gathered}
$$

If basis and test functions have opposite orientations, the following expression is obtained:

$$
\begin{aligned}
I_{2 b}= & \frac{1}{360}\left\{80 a^{5}+8 b^{5}-152 a^{5}+\left(72 a^{4}-8 b^{4}+4 a^{2} b^{2}\right)\right. \\
& \sqrt{a^{2}+b^{2}}+15 a b\left[-10 b^{3} \ln b+b\left(4 a^{2}+5 b^{2}\right)\right. \\
& \ln \left(-a+\sqrt{a^{2}+b^{2}}\right)+b\left(-4 a^{2}+5 b^{2}\right) \ln \left(a+\sqrt{a^{2}+b^{2}}\right) \\
& \left.\left.+6 a^{3} \ln \left(\frac{-b+\sqrt{a^{2}+b^{2}}}{b+\sqrt{a^{2}+b^{2}}}\right)\right]\right\} .
\end{aligned}
$$

Similar expressions are obtained when basis and test functions are oriented along other axes, just interchanging the dimensions $(a, b)$ by the dimensions along the relevant axes.

Finally, the volume-surface integral takes the following expression for the face shown in Fig. 1:

$$
\begin{aligned}
I_{5}= & \frac{1}{12}\left\{4 c^{4}+\left(6 a^{2} c-4 c^{3}\right) \sqrt{a^{2}+c^{2}}+\left(6 b^{2} c-4 c^{3}\right)\right. \\
& \sqrt{b^{2}+c^{2}}+\left(-6 a^{2} c-6 b^{2} c+4 c^{3}\right) d-24 a b c^{2} \arctan \left[\frac{a b}{c d}\right]
\end{aligned}
$$

$$
\begin{aligned}
& -8 a b^{3} \arctan \left[\frac{a c}{b d}\right]-8 a^{3} b \arctan \left[\frac{b c}{a d}\right] \\
& -2 a^{4} \ln a-2 b^{4} \ln b+\left(a^{4}-6 a^{2} b^{2}+b^{4}\right) \ln \left[a^{2}+b^{2}\right] \\
& +2 a^{4} \ln \left[c+\sqrt{a^{2}+c^{2}}\right]+2 b^{4} \ln \left[c+\sqrt{b^{2}+c^{2}}\right] \\
& -12 a b c\left(b \ln \left[\frac{-a+d}{a+d}\right]+a \ln \left[\frac{-b+d}{b+d}\right]\right) \\
& \left(-2 a^{4}+12 a^{2} b^{2}-2 b^{4}\right) \ln [c+d]+2 c^{3} \\
& \left(2 b\left(\ln \left[\frac{b+\sqrt{b^{2}+c^{2}}}{-b+\sqrt{b^{2}+c^{2}}}\right]+\ln \left[\frac{-b+d}{b+d}\right]\right)\right. \\
& +a\left(3 \ln \left[\frac{b^{2}+c^{2}}{c^{2}}\right]+\ln \left[\frac{-a+\sqrt{a^{2}+c^{2}}}{a+\sqrt{a^{2}+c^{2}}}\right]\right. \\
& \left.\left.+\ln \left[\frac{a+d}{-a+d}\right]+3 \ln \left[\frac{c^{2}+2 a\left(a+\sqrt{a^{2}+c^{2}}\right)}{b^{2}+c^{2}+2 a(a+d)}\right]\right)\right) \\
d= & \sqrt{a^{2}+b^{2}+c^{2}} .
\end{aligned}
$$

The integral for other faces can easily be obtained by interchanging $(a, b)$ by the new dimensions of the relevant face: $(a, c)$ or $(b, c)$. The volume-volume integrals are given in [8].

\section{REFERENCES}

[1] S. Makarov, S. Kulkarni, A. Marut, and L. Kempel, "Method of moments solution for a printed patch/slot antenna on a thin finite dielectric substrate using the volume integral equation," IEEE Trans. Antennas Propag., vol. 54, no. 4, pp. 1174-1184, Apr. 2006.

[2] A. F. Peterson, S. L. Ray, and R. Mittra, Computational Methods for Electromagnetics. Piscataway, NJ: IEEE Press, 1998, p. 451.

[3] F. Quesada Pereira, J. L. Gómez Tornero, D. Cañete Rebenaque, J. Pascual García, and A. Álvarez Melcón, "Analysis of finite microstrip structures using an efficient implementation of the integral equation technique," Radio Sci., vol. 40, no. RS1004, Jan. 2005.

[4] D. R. Wilton, S. M. Rao, A. W. Glisson, D. H. Schaubert, O. M. Al-Bundak, and C. M. Butler, "Potential integrals for uniform and linear source distributions on polygonal and polyhedral domains," IEEE Trans. Antennas Propag., vol. AP-32, no. 3, pp. 276-281, Mar. 1984.

[5] P. Arcioni, M. Bressan, and L. Perregrini, "On the evaluation of the double surface integrals arising in the application of the boundary integral method to 3-D problems," IEEE Trans. Microw. Theory Tech., vol. 45, no. 3, pp. 436-439, Mar. 1997.

[6] R. Cools, "Monomial cubature rules since Stroud: A compilation," J. Comput. Appl. Math., vol. 112, no. 1-2, pt. 2, pp. 21-27, 1999.

[7] P. R. Wellin, R. J. Gaylord, and S. N. Kamin, An Introduction to Programming with Mathematica, 3rd ed. Cambridge, U.K.: Cambridge Univ. Press, 2005.

[8] "Appendix: Final analytical expressions for the singular static integrals," Dec. 20, 2009 [Online]. Available: http://personales.upv.es/vboria/appendix.pdf 